\title{
Inappropriate Prescribing in Elderly Inpatients at a University Hospital in Saudi Arabia
}

\author{
Nisreen Abdulrahman Jastaniah ${ }^{1} \cdot$ Ahmed Saeed Almaqati $^{2} \cdot$ Anas Khaleel Alsuraihi $^{2} \cdot$ Sultan Adnan Abughanim ${ }^{2}$. \\ Mohammed Aseeri ${ }^{3}$
}

Published online: 7 September 2018

(c) The Author(s) 2018

\begin{abstract}
Background and objectives Elderly populations usually use more medications than any other age group and are therefore more susceptible to potentially inappropriate prescribing (PIP), drug-drug interactions, and the related health consequences. In this study, we aimed to determine PIP prevalence and explore the most common potentially inappropriate medications (PIMs) prescribed.

Methods This was a retrospective study involving elderly patients admitted at King Abdulaziz Medical City-Jeddah (KAMCJD), between November 2014 and January 2015. We included all elderly patients aged 60 years and above admitted to KAMC-JD through the emergency department (ED), clinic, and direct admissions during the study period. Patients admitted to the intensive care unit, oncology department, and/or those who passed way in the ED before admission were excluded from the study. Prescriptions were assessed for PIP using the 2012 Beers Criteria, which categorizes PIMs to three classes: first class are medications to avoid in older adults regardless of their conditions; second class are medications to avoid with certain diseases or syndromes; and third class are medications that should be used with caution.

Results Our study included 135 patients, of which $49.6 \%$ were males. The mean age was $71.26 \pm 8.1$ years. According to the 2012 Beers Criteria, $80 \%$ of patients were using at least one listed PIM. For the Criteria's first, second, and third classes, PIM prescription rates were $72.6 \%, 59.2 \%$, and $37.7 \%$, respectively. Regarding the most prescribed PIM in each class, insulin (sliding scale) was the most reported PIM in the first class, NSAIDs in the second class, and, lastly, vasodilators in the third class. Conclusion PIP is a serious health issue threatening elderly patients. There is a need to develop evidence-based, contextsensitive, and user-friendly tools to assess PIP, as well as supportive training programs.
\end{abstract}

Anas Khaleel Alsuraihi

Anas.k.s@hotmail.com

$1 \quad$ King Abdullah International Medical Research Center/King Saud Bin Abdulaziz University for Health Sciences, Family Medicine, Geriatric and Palliative Care, Ministry of National Guard-Health Affairs, Jeddah, Saudi Arabia

2 King Abdullah International Medical Research Center/King Saud Bin Abdulaziz University for Health Sciences College of Medicine, Ministry of National Guard-Health Affairs, Jeddah, Saudi Arabia

3 King Abdullah International Medical Research Center/King Saud Bin Abdulaziz University for Health Sciences, Ministry of National Guard-Health Affairs, Jeddah, Saudi Arabia

\section{Key Points}

PIP is major health burden for many societies; however, it is avoidable in most cases.

Awareness regarding Criteria for PIP is important, not only for physicians but for all stakeholders

\section{Introduction}

According to the World Health Organization (WHO), there is no one specific age that defines an individual elderly; however, the United Nation (UN) sets the age $60+$ years to refer to older populations [1]. Similarly, in the Kingdom of Saudi Arabia (KSA), many consider 60 years old and above the cutoff age to define an elderly individual. The number of 
older individuals is expected to rise in Saudi Arabia, and reach one-quarter of the total population by $2050[2,3]$. Elderly populations usually use more medications than any other age group, because of the many physical and functional changes related to the aging process, and the accompanying health problems. Thus, they are more susceptible to potentially inappropriate prescribing (PIP) as well as to drug-drug interactions [4-6].

PIP is becoming a major health burden, imposing serious threats to patients' health, and draining valuable health resources, which could be prevented by adopting the appropriate interventions [4]. Drug-related problems are quite common, especially among elderly populations suffering from multiple co-morbidities $[4,5]$. Potentially inappropriate medications (PIM) can be assessed using the American Geriatrics Society (AGS) Beers Criteria. These Criteria were developed to avoid inappropriate medication use in elderly patients, and prevent medication side effects and other drugrelated issues [6]. The Criteria are used to determine the prevalence of PIP and PIMs, since the global PIP prevalence ranges from 13 to 35\% [7]. Moreover, reported PIM usage rates were very variable across regions. Examples of lower rates were reported in Brazil [8] and Malaysia [9], 26.9\% and $32.7 \%$, respectively, while higher rates of $87.6 \%$ were reported in Spain [10]. Regional rates were also alarmingly high, even in developed countries. One study based on six European countries revealed an overall PIM prescription rate of $30.4 \%$ [11].

In Saudi Arabia, research focusing on this issue remains limited to primary-care settings where inpatients receive relatively little attention [12]. One study carried out in the Saudi Military Hospital in Riyadh in 2004 reported the PIP prevalence to be $2.1 \%$, with $43.6 \%$ of patients taking at least one PIM [13]. The latter highlights the need for more research exploring PIP rates, and PIM prescribed in the inpatient settings across different regions, especially with the growing elderly population in Saudi Arabia. PIP refers to prescribing by the physician of potentially inappropriate medications, while PIM refers to medications that have been identified through expert panel review as having an unfavorable balance of risks and benefits by themselves and considering the alternative treatments available. This study aimed to identify PIP, polypharmacy rates among inpatient elderly admitted to King Abdulaziz Medical CityJeddah (KAMC-JD), and explore the most common PIMs prescribed.

\section{Materials and Methods}

A retrospective review was conducted to identify the prevalence of PIP among elderly inpatients at King Khalid National Guard Hospital (KKNGH) in King Abdulaziz
Medical City-Jeddah (KAMC-JD), which was founded in July 1982. The hospital provides different medical services and specialties for the population in the Western Region. Its capacity for different medical services is approximately 480 beds. Ethical approval was obtained from the Research Ethics Review Board at King Abdullah International Medical Research Center, project number SP15/096. The review took place over 3 months, starting November 2014 and ending in January 2015. We targeted all elderly males and females aged 60 years and above who were admitted to KAMC-JD through an Emergency Department (ED), clinics, and direct admissions-including planned hospitalization-during the study period, and irrespective of their current prescriptions. Patients admitted to the intensive care unit, oncology department, and/or those who passed way in ED before admission were excluded from the study. A list of admitted patients was obtained periodically for the specific period of the study, and if the patient met the inclusion Criteria s/he was reviewed from admission to 1 month or until discharge. Therefore, we preferred the retrospective design although it has some elements of prospective follow-up.

The sample size was calculated using a $95 \%$ confidence interval and a 5\% margin of error, and estimated a sample of 135 patients. We used a non-probability convenient sampling technique to select our sample from the above group. Since we only reviewed electronic patient files to extract relevant data, no consent was needed; however, all personal data including name, contact details, and diagnosis remained confidential.

Prescriptions were assessed for PIP according to the 2012 Beers Criteria. It has been the most commonly consulted reference for assessing medication prescriptions in elderly patients for over 20 years [6]. The Criteria categorize PIMs to three classes: First Class: medications to avoid in older adults regardless of their conditions. Second Class: medications to avoid with certain diseases or syndromes. Third Class: medications that should be used with caution $[6,13]$. Polypharmacy has many classifications; however, in our study, we considered "taking five medications or moreincluding pro re nata (PRN, when needed)" as polypharmacy [14].

We used a standardized data extraction sheet to collect relevant information from patient medical records (files and Quadramed System). The sheet had two main sectionspatient demographics and medication profile. Demographic data included serial coding number, age, gender, diagnosis on admission, date of admission, and co-morbidities/medical conditions (all co-morbidities in the Charlson co-morbidity index were accounted for in each patient; since this was not an objective for our research, little attention was given to this) [15], and the date of discharge. The medication profile during admission focused on current prescriptions, their dose, frequency, and route of administration involving all 
medications administered to the patients and even prescribed medication that was not given. The data extraction tool was piloted, and tools were used for face/content validity. Finally, the data were then peer-reviewed by two assessors to examine other characteristics. Medical interns involved in reviewing patient medication profiles were introduced to inappropriate prescribing, and intensively trained on the Beers Criteria by a geriatrician and clinical pharmacist. During data collection, data were coded, crosschecked, and then entered and saved in a protected device.

For analysis, data were entered and analyzed using IBM Statistical Package for Social Sciences (SPSS) version 20. Simple descriptive statistics were used to define the characteristics of study variables. While counts and percentages were used for categorical and nominal variables, continuous variables were presented as means and standard deviations. For the comparative analysis, we used a Chi square test and $t$ test for qualitative and quantitative data, respectively. A p-value of $<0.05$ was considered significant.

\section{Results}

For this review 135 patients were included, and out of those 67 (49.6\%) were male. The mean age was $71.26 \pm 8.1$ years, and patients were classified into the following three age groups: $60-75$ years, $76-85$ years, and $>85$ years, representing $70.4 \%, 24.4 \%$, and $5.2 \%$ of the cohort, respectively. On average, patients spent $20.5 \pm 23$ days in hospital during their present admission and suffered from $4.3 \pm 2.4$ co-morbidities. Furthermore, polypharmacy was detected in $93.3 \%$ of patients (Table 1).

Regarding prescription profiles, $80 \%$ of our patients were prescribed at least one medication listed in the 2012 Beers Criteria. On the univariate analysis we discovered that PIP rates were significantly higher in patients with a hospital stay exceeding 30 days $(n=26, p=0.007)$. Our data showed that PIP is higher in patients with polypharmacy, for example those patients currently taking five or more medications $(p<0.001)$. On the other hand, we found no association between high PIP rates and gender, different age groups, or type of clinical ward.

For PIM prescription, and per the first class of Beers Criteria, PIMs were found in $72.6 \%$ of patients, and $27.4 \%$ used only one medication (Fig. 1). The most common PIM prescribed in the first class was insulin, with a sliding scale used in $56(41.5 \%)$ patients followed by metoclopramide taken by 49 patients (36.3\%). Forty patients of those on insulin and 34 of the metoclopramide users were within the first age group, i.e., the younger groups. Eighty patients (59.2\%) were at least on one PIM of the second class of Beers Criteria (Fig. 1). Non-steroidal anti-inflammatory drugs (NSAIDs) were the most commonly prescribed PIMs, and were used
Table 1 General characteristics of the patients included in the study

\begin{tabular}{|c|c|c|c|}
\hline Characteristic & $\begin{array}{l}\text { Total } \\
135\end{array}$ & $\begin{array}{l}\text { Male } \\
67\end{array}$ & $\begin{array}{l}\text { Female } \\
68\end{array}$ \\
\hline \multicolumn{4}{|l|}{ Age, years } \\
\hline Mean $\pm S^{a}$ & $71 \pm 8$ & $72 \pm 8.4$ & $70 \pm 7.6$ \\
\hline $60-75^{\mathrm{b}}$ & $95(70.4)$ & $42(62.7)$ & $53(77.9)$ \\
\hline $76-85^{\mathrm{b}}$ & $33(24.4)$ & $23(34.4)$ & $10(14.7)$ \\
\hline$>85^{\mathrm{b}}$ & $7(5.2)$ & $2(2.9)$ & $5(7.4)$ \\
\hline \multicolumn{4}{|l|}{ Co-morbidity } \\
\hline No. of co-morbidities ${ }^{\mathrm{a}}$ & $4.3 \pm 2.4$ & $4.4 \pm 2.2$ & $4.1 \pm 2.6$ \\
\hline \multicolumn{4}{|l|}{ Polypharmacy } \\
\hline Present $^{\mathrm{b}}$ & $126(93.3)$ & $61(91.1)$ & $65(95.6)$ \\
\hline Absent ${ }^{b}$ & $9(6.7)$ & $6(8.9)$ & $3(4.4)$ \\
\hline $\begin{array}{l}\text { Mean } \pm \mathrm{SD}^{\mathrm{a}} \text { of regular } \\
\text { and PRN medications }\end{array}$ & $19 \pm 10$ & $18.2 \pm 9.7$ & $19.7 \pm 9.8$ \\
\hline \multicolumn{4}{|c|}{ Duration of hospitalization } \\
\hline Mean $\pm S^{a}$ & $20.5 \pm 23$ & $22 \pm 28$ & $19 \pm 17$ \\
\hline$\leq 30$ days $^{\mathrm{b}}$ & $109(80.7)$ & $52(77.6)$ & $57(83.8)$ \\
\hline$>30$ days $^{\mathrm{b}}$ & $26(19.3)$ & $15(22.4)$ & $11(16.2)$ \\
\hline
\end{tabular}

${ }^{\mathrm{a}}$ Mean \pm standard deviation

${ }^{\mathrm{b}} n(\%)$

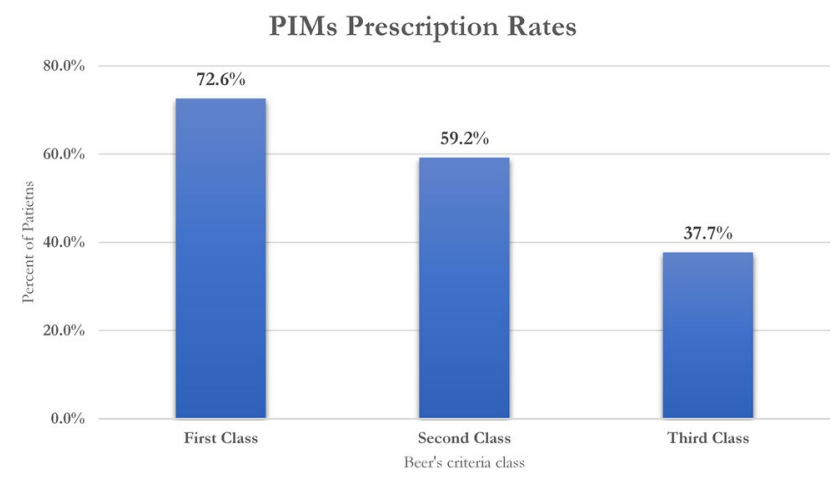

Fig. 1 PIM prescription rates within the three classes of the 2012 Beers Criteria

by 14 patients (17.5\%) with the following diagnoses: chronic kidney disease (stages IV and V), heart failure, and those with a positive history of a gastric or duodenal ulcer. For this class, the PIM prescription rate was higher in patients admitted to the surgical and medical wards $(p<0.001)$. However, there is no significant difference between groups when compared by gender, age, length of hospital stay, or polypharmacy. Finally, for the third class we found that 51 (37.7\%) patients were prescribed at least one medication in this class (Fig. 1), with vasodilators as the most commonly prescribed drug with a $24.4 \%$ prescription rate. PIM prevalence was higher in patients admitted to both surgical and medical wards $(\mathrm{p}<0.001)$. Table 2 illustrates the most commonly encountered PIMs according to Beers criteria. 
Table 2 The most commonly encountered PIPs as per the 2012 Beers Criteria

\begin{tabular}{|c|c|}
\hline PIMs & $\begin{array}{l}\text { Frequency } \\
N(\%)\end{array}$ \\
\hline \multicolumn{2}{|l|}{ First class } \\
\hline Insulin, sliding scale & $56(41.5 \%)$ \\
\hline Metoclopramide & $49(36.3 \%)$ \\
\hline Diphenhydramine & $31(23 \%)$ \\
\hline Lorazepam & $16(11.9 \%)$ \\
\hline $\begin{array}{l}\text { First- and second-generation antipsychotics (haloperidol, trifluoperazine, aripiprazole, olan- } \\
\text { zapine, quetiapine, risperidone) }\end{array}$ & $16(11.9 \%)$ \\
\hline Nifedipine, immediate-release & $10(7.4 \%)$ \\
\hline \multicolumn{2}{|l|}{ Second class } \\
\hline \multicolumn{2}{|l|}{ NSAIDs and COX-2 inhibitors } \\
\hline \multicolumn{2}{|l|}{ Use among patients with heart failure } \\
\hline \multicolumn{2}{|l|}{ Use among patients with history of gastro/duodenal ulcer } \\
\hline $\begin{array}{l}\text { NSAIDs } \\
\text { Use among patients with CKD stages IV and V }\end{array}$ & $11(8.5 \%)$ \\
\hline \multicolumn{2}{|l|}{ Use among patients with LUTS, or benign prostatic hyperplasia } \\
\hline $\begin{array}{l}\text { Anticonvulsants } \\
\text { Use among patients with a history of falls or fractures }\end{array}$ & $4(3 \%)$ \\
\hline Benzodiazepines & $2(1.5 \%)$ \\
\hline TCAs and SSRIs & $2(1.5 \%)$ \\
\hline $\begin{array}{l}\text { Antiemetics } \\
\text { Metoclopramide, prochlorperazine, promethazine }\end{array}$ & $2(1.5 \%)$ \\
\hline \multicolumn{2}{|l|}{ Use among patients with Parkinson's disease } \\
\hline \multicolumn{2}{|l|}{ Third class } \\
\hline Vasodilators & $33(24.4 \%)$ \\
\hline Antipsychotics & $12(8.9 \%)$ \\
\hline Dabigatran & $1(0.7 \%)$ \\
\hline
\end{tabular}

NSAIDs non-steroidal anti-inflammatory drugs, $C O X-2$ cyclooxygenase-2, TCAs tricyclic antidepressants, SSRIs selective serotonin reuptake inhibitors, LUTS lower urinary tract syndrome, $C K D$ chronic kidney disease

\section{Discussion}

When comparing our rates of PIP to other regions in Saudi Arabia, they are considered generally higher [13]; however, the lack or unsuitability of guidelines to local settings can increase the gravity of this situation across the kingdom, and highlights the need for quick action in this regard. Moreover, the lower rates reported elsewhere in Saudi Arabia (e.g., in Al-Riyadh) were focused mainly on outpatient groups, which are usually more stable and healthier populations. Also, previous reports are based on an older version of Beers Criteria, namely the 2003 version, and hence some PIMs may have been missed [13]. It must be acknowledged that Beers Criteria were originally established to detect potentially inappropriate prescribing; therefore, increasing PIP rates and using PIMs do not necessitate the acknowledgment of adverse effects, and/or existence of complications, and therefore close clinical follow-up, by specialized geriatric teams, and more focused research on PIP interactions is crucial.

With regard to the literature, similar high PIM rates were reported from different populations within the region. One study conducted in Taiwan revealed a PIM rate of $86.2 \%$ among the geriatric patients in ambulatory care centers [17]. Every patient was on at least one PIM, and an NSAID was the most common PIM prescribed, corresponding to our results. However, for the Taiwanese group, being young was associated with higher rates of PIP. These rates were attributed to the governmental modalities in Taiwan, aiming to reduce drug expenditure. In addition to providing drugs freely in governmental hospitals, most of the prescribed medications were generally cheaper drugs such as non-cyclooxygenase-2 (Cox2) inhibitor NSAIDs. Another factor was the unrestricted accessibility to both specialized 
physicians and drug purchasing, without the need for primary-care consultations [17]. All three factors could also apply to our situation, despite the differences in patient characteristics. Another regional study that focused on geriatric residents of Malaysian nursing homes [9], related their high PIP rates to drug availability and zero costs, especially since centers were sponsored governmentally or were donation dependent. The study also held the lack of specialized geriatric consultations and services responsible [9]. Geriatric teams must include clinical pharmacists, who are involved in the prescription process. Adopting computerized alert systems and encouraging comprehensive and thorough documentation of patient co-morbidities, medication profiles, and functional status could decrease PIP incidents in similar facilities.

On the other hand, studies from other regions presented lower rates of PIP, and differences among patients experiencing most of the PIP incidents. A large national study in the USA found the prevalence of PIP within their population to be $42.6 \%$, and NSAIDs and benzodiazepine were the most commonly prescribed PIMs, at $10.9 \%$ and $9.3 \%$, respectively [16]. These lower rates coincide with the large diverse sample involved $(n=18,475)$ in this study, recruited from different health-care facilities. Another factor that can explain lower rates is the greater familiarity among physicians with AGS Beers Criteria, and resulting in more implementation [16]. Finally, and unlike our systems, their medical information records cannot differentiate between the different insulin regimens, and therefore insulin sliding scales were excluded from their analysis, possibly missing important PIP incidents. Another example is a Brazilian study with a PIP prevalence of $26.9 \%$. Despite corresponding to some of our results, such as an increased number of medications associated with higher PIP rates, unlike our population, this group showed a probable association between being a female and experiencing PIP [8].

One example from Europe is a study from Belgium in elderly patients visiting primary health-care centers, who had lower PIP and polypharmacy rates, at $32 \%$ and $61 \%$, respectively. The latter differences could be explained by the limited variation in age groups in the Belgium study, which included patients aged 80 years and above only [18]. Non-benzodiazepine hypnotics were generally the most commonly used PIM in $6.2 \%$ of residents. Aspirin was most commonly prescribed third-class PIM per Beers Criteria, to be used with caution, in contrast to our study where vasodilators were the most commonly used. In New Zealand PIM dispensing was identified in $40.39 \%$ of the total elderly population [19], universally lower than rates reported in our study. Taking into consideration the large national scale-inclusiveness in the New Zealand study, it was also acknowledged that some uncovered and over-the-counter medicines may have not been included in the analysis, and thus it may have underestimated patient exposure to PIMs [19]. Finally, a recently published article in 2014 evaluated PIMs in patients admitted to residential older age care facilities in Australia, and reported that at least one PIM was used by $54.4 \%$ of residents on admission, and by $49.5 \%$ when discharged [20]. The authors emphasized that these admissions were considered wasted opportunities to review patient medications and minimize chances for PIP. These researchers as well as Al-Dhawailie's report advocated for developing more sensitive tools, e.g., applying technology and computerized physician orders to better capture PIP cases, and allow for more involvement of both geriatricians and pharmacists in prescription processes [20,21].

This review revealed a relatively high prevalence of PIP and polypharmacy among our elderly patients. This could be explained by and attributed to several factors. First the study site itself should be considered; the research was conducted in a tertiary-care hospital, known to receive numerous and complex referred cases from other hospitals in the western region of Saudi Arabia, and thus the studied population included severely sick patients with multiple co-morbidities, with an average of four diagnosed conditions per patient. As expected, these patients were on several medications. In order to enhance the research in this area and better inform health policies, future studies should include more than one facility at different levels of care, which will help minimize bias when recruiting patients and selecting a more nationally representative sample.

One limitation to this review is that Beers Criteria were established in the USA, and not all medications listed are currently available in our institution; in addition there are differences between countries regarding patient care pathways and accessibility. Furthermore, we could not test the relationship between PIP, drug-drug interactions, and their consequences, and while the review included 12 patients who passed away during their hospital stay, we cannot relate their death to PIP or prescription of PIMs. In addition, we could not illustrate differences between "when needed" and regular medications. More research is warranted to make such judgments. Also, it must be acknowledged that due to time and resources constraints, our study was conducted in a single tertiary-care center.

Additionally, the lack of local working guidelines assessing PIP and regulating the use of PIMs, plus the lack of professional healthcare awareness on the international criteria (e.g., Beers) could be responsible for these rates. Even when introducing healthcare providers to guidelines such as Beers or START STOPP, they could remain inapplicable to local settings when comparing drug availabilities, alternatives, and patient preferences. Finally, we recommend that future studies involve more categorization of patients and analysis, one example being to assess the difference between planned and unplanned admissions. 


\section{Conclusion}

PIP is a serious health issue threatening elderly patients who are presenting to hospitals in increasing numbers. There is a need to develop an evidence-based, context-sensitive, and user-friendly tool to assess PIP, as well as supportive training programs for specialized teams involving geriatricians and clinical pharmacists.

\section{Compliance with Ethical Standards}

Funding No external funding was recieved to conduct this research.

Conflicts of Interest All authors have no conflicts in interest to declare.

Open Access This article is distributed under the terms of the Creative Commons Attribution-NonCommercial 4.0 International License (http://creativecommons.org/licenses/by-nc/4.0/), which permits any noncommercial use, distribution, and reproduction in any medium, provided you give appropriate credit to the original author(s) and the source, provide a link to the Creative Commons license, and indicate if changes were made.

\section{References}

1. World Health Organization. Proposed working definition of an older person in Africa for the MDS project. 2013. Definition of an older or elderly person. http://www.who.int/healthinfo/survey/ ageingdefnolder/en/ Accessed Feb 2015.

2. Abusaaq H I. Population aging in Saudi Arabia. SAMA working paper. 2015.

3. Alsulami MH, Anthony SA. A model of a proactive ambient assisted living system to monitor elderly people in The Kingdom of Saudi Arabian. IJRET. 2016;4:2277-4378.

4. Hines LE, Murphy JE. Potentially harmful drug-drug interactions in the elderly: a review. Am J Geriatr Pharmacother. 2011;9:364-77.

5. Goldberg RM, Mabee J, Chan L, Wong S. Drug-drug and drugdisease interactions in the ED: analysis of a high-risk population. Am J Emerg Med. 1996;14:447-50.

6. Fick DST, Beizer J, Brandt N, Dombrowski R, DuBeau CE, Flanagan N, et al. American Geriatrics Society updated Beers Criteria for potentially inappropriate medication use in older adults. J Am Geriatr Soc. 2012;60:616-31.

7. Lam MP, Cheung BM, Wong IC. Prevalence of potentially inappropriate prescribing among Hong Kong older adults: a comparison of the beers 2003, beers 2012, and screening tool of older person's prescriptions and screening tool to alert doctors to right treatment criteria. J Am Geriatr Soc. 2015;63:1471-2.

8. Faustino CG, Passarelli MC, Jacob-Filho W. Potentially inappropriate medications among elderly Brazilian outpatients. Sao Paulo Med J. 2013;131:19-26.

9. Chen LL, Tangiisuran B, Shafie AA, Hassali MA. Evaluation of potentially inappropriate medications among older residents of Malaysian nursing homes. Int J Clin Pharm. 2012;34:596-603.

10. San-José A, Agustí A, Vidal X, Formiga F, López-Soto A, Fernández-Moyano A, et al. Inappropriate prescribing to older patients admitted to hospital: a comparison of different tools of misprescribing and underprescribing. Eur J Intern Med. 2014;25:710-6.

11. Gallagher P, Lang PO, Cherubini A, Topinková E, Cruz-Jentoft A, Errasquín BM, et al. Prevalence of potentially inappropriate prescribing in an acutely ill population of older patients admitted to six European hospitals. Eur J Clin Pharmacol. 2011;67:1175-88.

12. Mahmoud MA, Aljadhey H, Hassali MA. Prescribing errors incidence in hospitalized Saudi patients: methodology considerations. SPJ. 2014;22(4):388-9.

13. Al-Omar HA, Al-Sultan MS, Abu-Auda HS. Prescribing of potentially inappropriate medications among the elderly population in an ambulatory care setting in a Saudi military hospital: trend and cost. Geriatr Gerontol int. 2013;13:616-21.

14. Masnoon N, Shakib S, Kalisch-Ellett L, Caughey GE. What is polypharmacy? A systematic review of definitions. BMC Geriatrics. 2017;17:230. https://doi.org/10.1186/s12877-017-0621-2.

15. Quan H, Li B, Couris CM, Fushimi K, Graham P, Hider P, et al. Updating and validating the charlson comorbidity index and score for risk adjustment in hospital discharge abstracts using data from 6 countries. Am J Epidemiol. 2011;173(6):676-82.

16. Bushardt RL, Massey EB, Simpson TW, Ariail JC, Simpson KN. Polypharmacy: misleading, but manageable. Clin Interv Aging. 2008;3:383.

17. Chang CB, Yang SY, Lai HY, Wu RS, Liu HC, Hsu HY, et al. Application of three different sets of explicit Criteria for assessing inappropriate prescribing in older patients: a nationwide prevalence study of ambulatory care visits in Taiwan. BMJ Open. 2015;5:e008214.

18. Dalleur O, Boland B, De Groot A, Vaes B, Boeckxstaens P, Azermai $\mathrm{M}$, et al. Detection of potentially inappropriate prescribing in the very old: cross-sectional analysis of the data from the BELFRAIL observational cohort study. BMC geriatrics. 2015;15:156.

19. Nishtala PS, Bagge ML, Campbell AJ, Tordoff JM. Potentially inappropriate medicines in a cohort of community-dwelling older people in New Zealand. Geriatr Gerontol Int. 2014;14:89-93.

20. Poudel A, Peel NM, Nissen L, Mitchell C, Gray LC, Hubbard RE. Potentially inappropriate prescribing in older patients discharged from acute care hospitals to residential aged care facilities. Ann Pharmacother. 2014;48:1425-33.

21. Al-Dhawailie AA. Inpatient prescribing errors and pharmacist intervention at a teaching hospital in Saudi Arabia. SPJ. 2011;19(3):193-6. 